\title{
Surface modification of reverse osmosis membranes for enhanced boron removal and fouling resistance
}

Jinmei Li, Dong Wang, ${ }^{*}$ Yang Wang, Baoyu Gao, ${ }^{*}$ and Zhining Wang ${ }^{*}$

Shandong Provincial Key Laboratory of Water Pollution Control and Resource Reuse,

School of Environmental Science and Engineering, Shandong University, Qingdao 266237, P. R. China

*Corresponding author: wangzhn@sdu.edu.cn and bygao@sdu.edu.cn 


\section{Supporting Information}

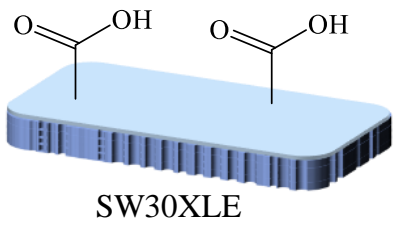

EDC/NHS

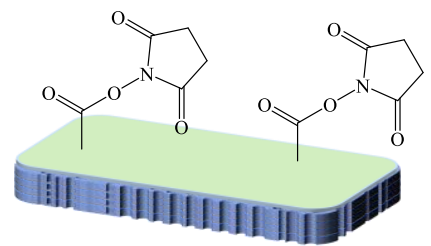

TOB:

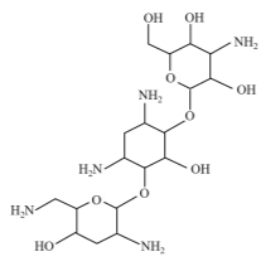

APD:
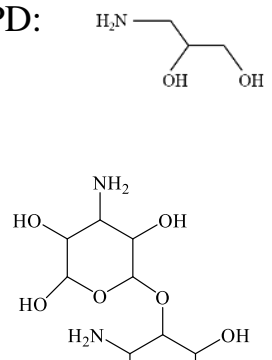

\section{APD/TOB}

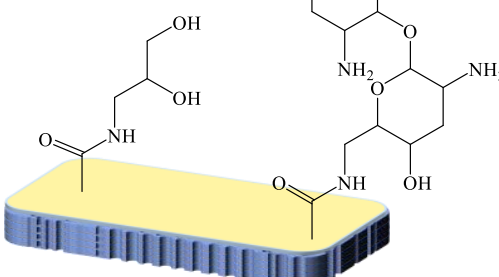

Scheme S1. Schematics of membrane grafting approaches.

Table S1 Elemental composition of the membranes, APD, and TOB molecules.

\begin{tabular}{|c|c|c|c|c|c|}
\hline \multirow{2}{*}{ Membranes } & \multicolumn{3}{|c|}{ Atomic concentration (\%) } & \multicolumn{2}{|c|}{ Atomic ratio } \\
\hline & $\mathrm{C}(1 \mathrm{~s})$ & $\mathrm{O}(1 \mathrm{~s})$ & $\mathrm{N}(1 \mathrm{~s})$ & $\mathrm{O} / \mathrm{N}$ & $\mathrm{O} / \mathrm{C}$ \\
\hline APD & 50.00 & 33.33 & 16.67 & 2.00 & 0.66 \\
\hline TOB & 56.25 & 28.13 & 15.62 & 1.80 & 0.50 \\
\hline SW30XLE & 77.00 & 10.26 & 12.74 & 0.81 & 0.13 \\
\hline TFC-TOB $2 / \mathrm{APD}_{0}$ & 75.03 & 12.58 & 12.39 & 1.02 & 0.17 \\
\hline TFC-TOB $0 / \mathrm{APD}_{10}$ & 76.28 & 12.21 & 11.51 & 1.06 & 0.16 \\
\hline $\mathrm{TFC}^{\mathrm{TOB}} \mathrm{B}_{2} / \mathrm{APD}_{10}$ & 75.33 & 12.81 & 11.86 & 1.08 & 0.17 \\
\hline
\end{tabular}

Table S2 List of isotherm parameters for boron adsorption of TFC-TOB $/ \mathrm{APD}_{10}$ membrane.

\begin{tabular}{cccccccc}
\hline & \multicolumn{3}{c}{ Langmuir constants } & \multicolumn{4}{c}{ Freundlich constants } \\
\cline { 2 - 7 } Membrane & $q_{\max }(\mathrm{mg} / \mathrm{g})$ & $\begin{array}{c}K_{L} \\
(\mathrm{~g} / \mathrm{mg})\end{array}$ & $R^{2}$ & $\begin{array}{c}K_{f} \\
\left((\mathrm{mg} / \mathrm{g})(\mathrm{L} / \mathrm{mg})^{1 / n}\right)\end{array}$ & $1 / n$ & $R^{2}$ \\
\hline $\begin{array}{c}\mathrm{TFC}- \\
\mathrm{TOB}_{2} / \mathrm{APD}_{10}\end{array}$ & 2.052 & 0.04 & 0.9795 & 0.191 & 0.50 & 0.9459 \\
\hline
\end{tabular}




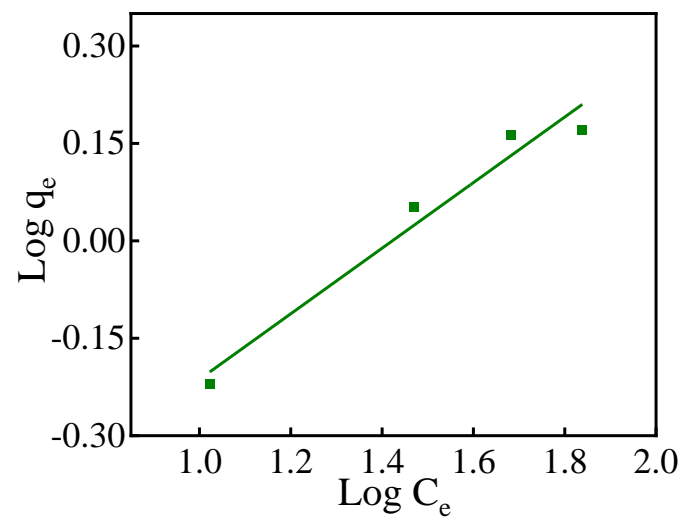

Figure S1. Freundlich adsorption isotherm of TFC-TOB $2 / \mathrm{APD}_{10}$ membrane.
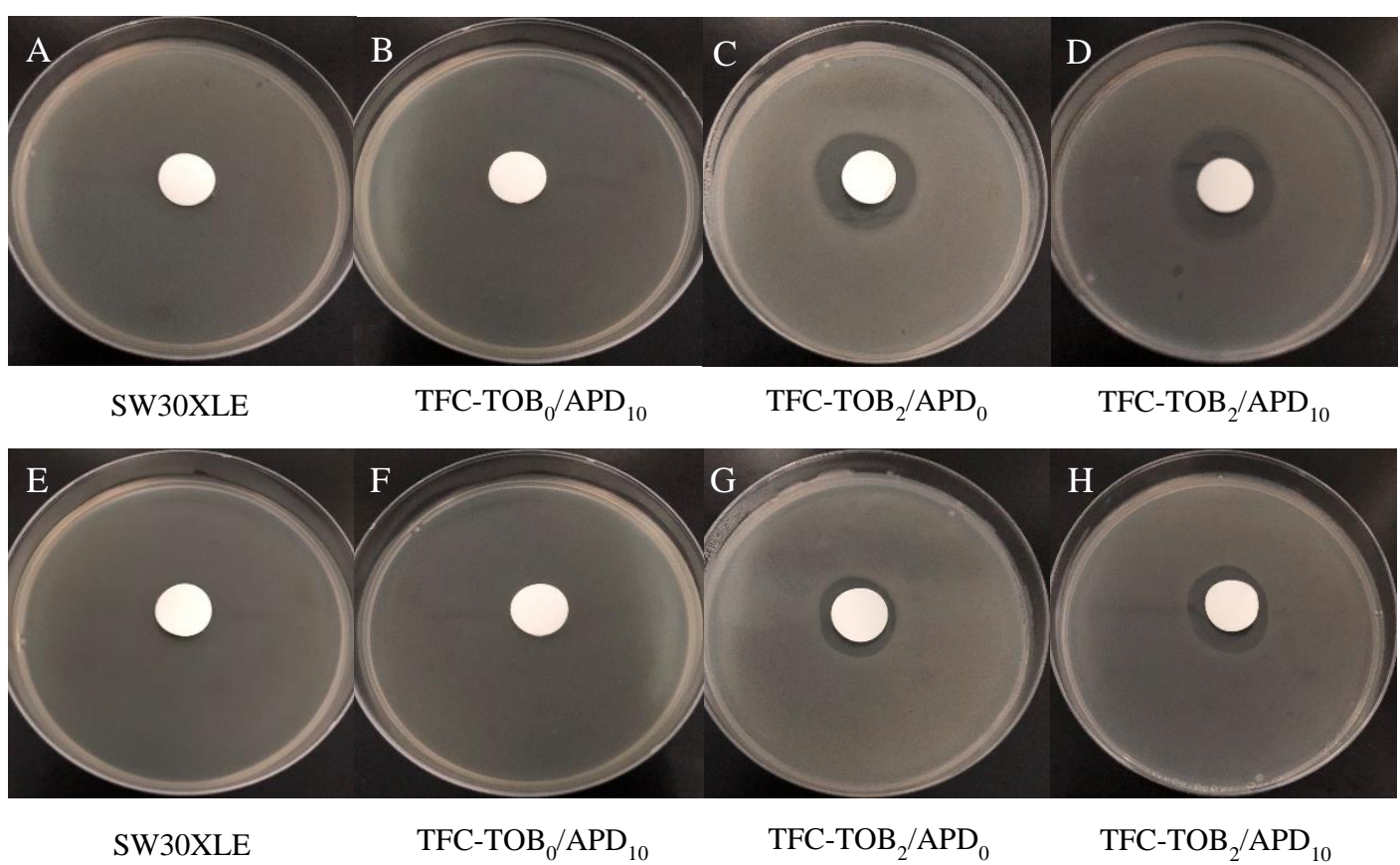

Figure S2. Diffusion inhibition zone tests for gram-negative E. coli (A D) and grampositive $S$. aureus $(\mathrm{E} \sim \mathrm{H})$. 


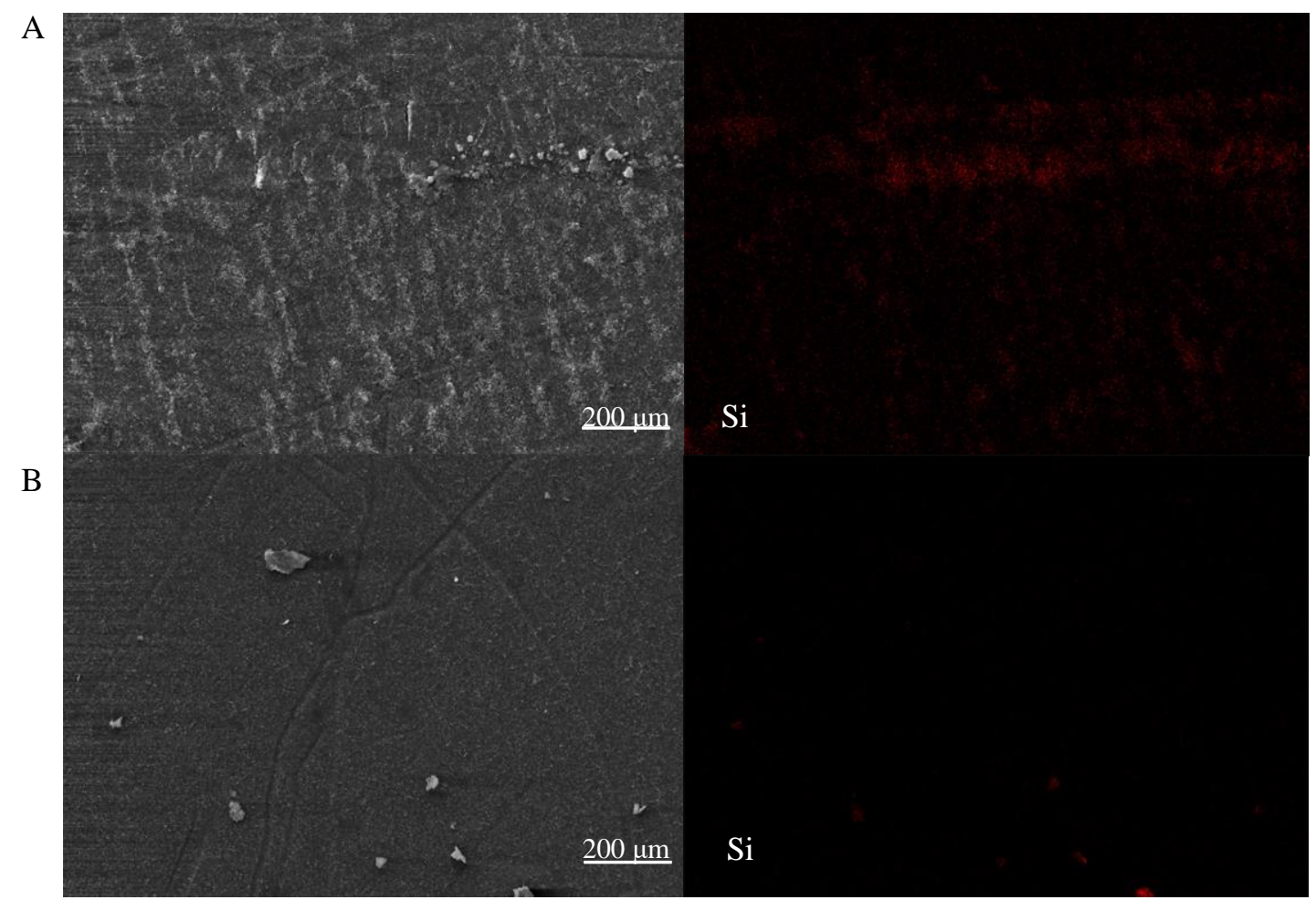

Figure S3. SEM and EDS mapping images of SW30XLE membrane and TFC$\mathrm{TOB}_{2} / \mathrm{APD}_{10}$ membrane after scaling test.

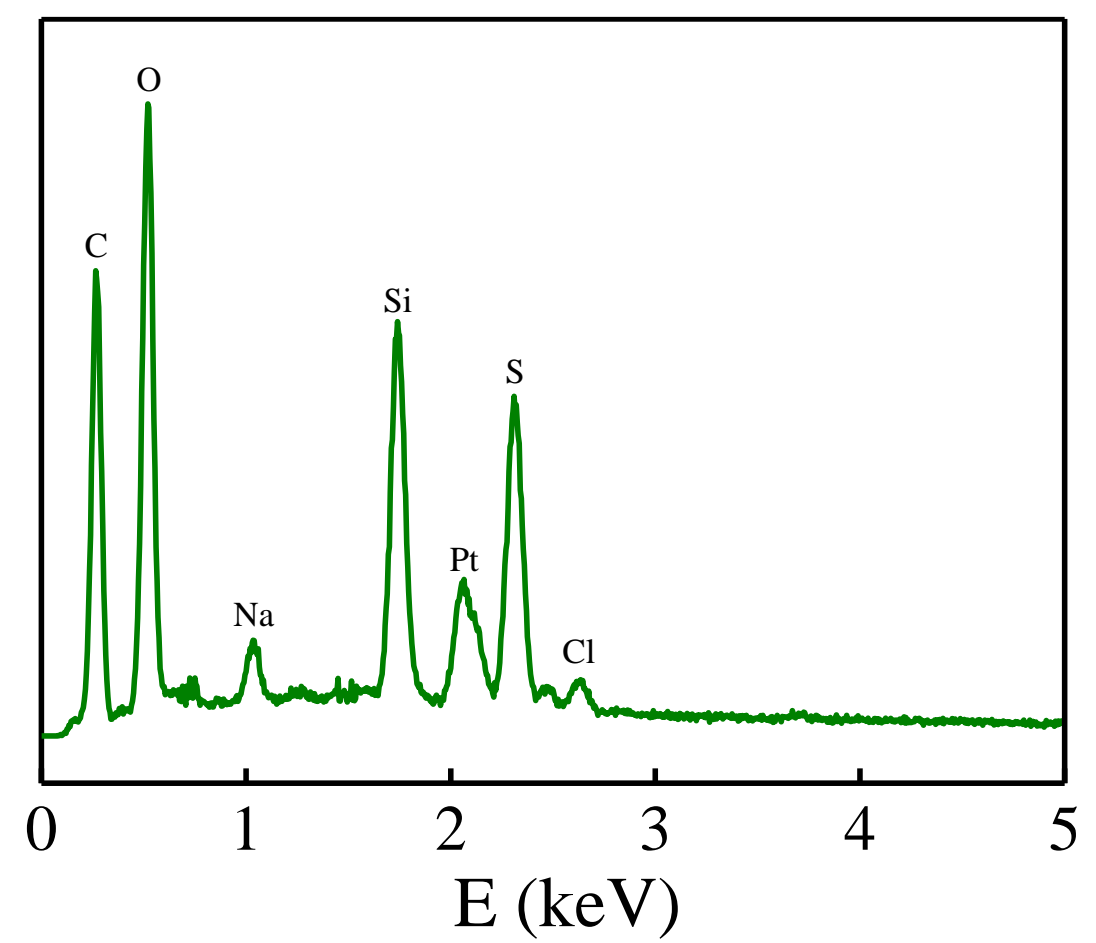

Figure S4. EDX image of the scaling substances on the surface of SW30XLE membrane. 